\title{
Coronary vasospasm caused by local infiltration of epinephrine after spinal anesthesia
}

\author{
Mi Geum Lee ${ }^{1}$, Hee Yeon Park ${ }^{1}$, Chang Ki Lee ${ }^{2}$, Joon Hyouk Choi ${ }^{3}$, and Yun Suk Choi ${ }^{4}$ \\ ${ }^{1}$ Department of Anesthesiology and Pain Medicine, Gachon University Gil Hospital, Incheon, ${ }^{2}$ Jeju National University School of \\ Medicine, ${ }^{3}$ Division of cardiology, Internal Medicine, Jeju National University Hospital, ${ }^{4}$ Department of Anesthesiology and Pain \\ Medicine, Jeju National University Hospital, Jeju, Korea
}

Local infiltration of epinephrine at the surgical site has been widely used to improve the operating view, due to reduced blood loss caused by vasoconstriction [1]. However, the use of epinephrine can cause various adverse effects such as severe hypertension, ventricular arrhythmia, myocardial infarction, cardiomyopathy, cardiac arrest, and acute pulmonary edema [1]. Although there have been some cases of epinephrine-induced cardiovascular events with therapeutic intravenous administration or local infiltration under general anesthesia [1-5], there were no previous reports of coronary vasospasm following local infiltration of epinephrine after spinal anesthesia confirmed by immediate coronary angiography. The anesthetic management of this condition is presented along with a review of the literature.

A 46-year-old woman (height $161 \mathrm{~cm}$, weight $60 \mathrm{~kg}$ ) entered the hospital to undergo a tension-free vaginal tape operation for stress incontinence. She had no previous medical history. Preoperative electrocardiogram (ECG), chest X-ray, and blood chemistry were found to be normal. Preoperative vital signs were: blood pressure (BP) 114/66 mmHg, heart rate (HR) 74 beats/ min, oxygen saturation $\left(\mathrm{SpO}_{2}\right) 99 \%$.

We gave her spinal anesthesia with $0.5 \%$ bupivacaine (Marcaine $^{\circledR}$, AstraZeneca AB, Södertälje, Sweden) $10 \mathrm{mg}$ intrathecally. Five minutes later, the anesthetic level reached T6, the patient was placed in the lithotomy position, and we then provided $5 \mathrm{~L} / \mathrm{min}$ of oxygen using a facial mask with a reservoir.

Fifteen minutes later, the surgery was initiated and the patient complained of headache and began to be restless. An ECG showed tachyarrhythmia, and the $\mathrm{HR}$ and $\mathrm{BP}$ rose to 160 beats/min and 210/130 mmHg, respectively. Esmolol $30 \mathrm{mg}$ was instantly injected intravenously (IV), but the patient's vital signs did not change. She complained of anxiety with sweating, and midazolam $2 \mathrm{mg}$ was IV injected. Through communication with the surgeon, we realized that epinephrine $1 \mathrm{mg}$ diluted with normal saline $10 \mathrm{ml}$ had been injected subcutaneously (SC) at the beginning of the surgery. Immediately, we administrated thiopental $200 \mathrm{mg}$ and rocuronium $30 \mathrm{mg} \mathrm{IV}$, and a laryngeal mask airway (LMA) \#4 was placed. Anesthesia was maintained with an air- $\mathrm{O}_{2}$ mixture $\left(\mathrm{FIO}_{2} 0.5\right)$ and end-tidal sevoflurane $1.0 \mathrm{vol} \%$, and we performed arterial catheterization to allow continuous monitoring of BP.

Twenty-five minutes after the induction of anesthesia, the patient's BP decreased to $78 / 48 \mathrm{mmHg}$, and ephedrine $5 \mathrm{mg}$ was IV injected. Immediately, the BP increased to $85-125 / 50-80$ $\mathrm{mmHg}$, and an ECG showed restoration of normal sinus rhythm. Therefore, the surgery was begun, and the patient maintained a BP of 84-120/62-78 mmHg and HR of 88-95 beats/min. The surgery was completed without incident. We injected $0.4 \mathrm{mg}$ of glycopyrrolate and $1.5 \mathrm{mg}$ of neostigmine IV, confirmed the patient's spontaneous breathing, and removed the LMA.

After arrival in the recovery room, the patient's $\mathrm{SpO}_{2}$ was $100 \%$ and $\mathrm{HR}$ was 81 beats/min, but the BP decreased to $75 / 44$ $\mathrm{mmHg}$. After two injections of ephedrine $5 \mathrm{mg}$ IV, the BP increased to $93-108 / 61-75 \mathrm{mmHg}$, but the patient complained of

Corresponding author: Yun Suk Choi, M.D., Ph.D., Department of Anesthesiology and Pain Medicine, Jeju National University Hospital, Aran 13gil 15(Ara-1Dong), Jeju 690-767, Korea. Tel: 82-64-750-1260, Fax: 82-64-750-1166, E-mail: solafide5@yahoo.co.kr

(c) This is an open-access article distributed under the terms of the Creative Commons Attribution Non-Commercial License (http:// creativecommons.org/licenses/by-nc/3.0/), which permits unrestricted non-commercial use, distribution, and reproduction in any medium, provided the original work is properly cited. 

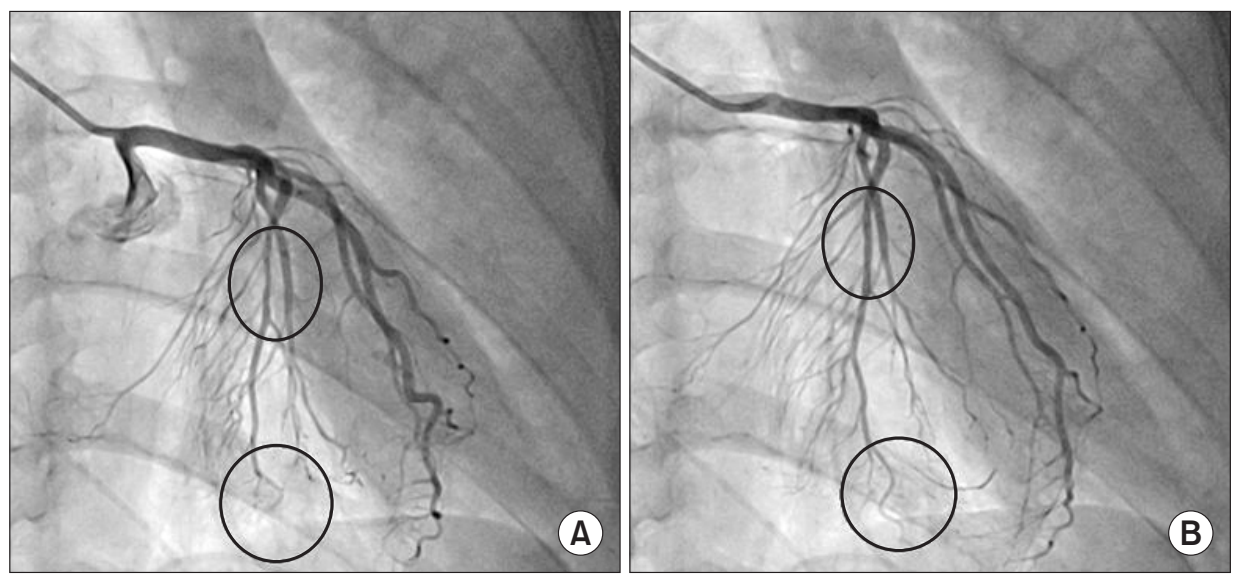

Fig. 1. Emergency coronary angiography shows a filling defect in the distal LAD and compression in the mid-LAD (A), which is relieved by intracoronary nitroglycerin injection (B). LAD indicates left anterior descending coronary artery. chest pain. An ECG showed ST segment depression in leads II, III, and aVF. The blood levels of creatine kinase MB and troponin T were found to be $6.9 \mathrm{ng} / \mathrm{ml}$ and $0.347 \mathrm{ng} / \mathrm{ml}$, respectively. Emergency coronary angiography showed a spasm of the left anterior descending coronary artery (LAD), and coronary artery expansion was confirmed following injection of $200 \mu \mathrm{g}$ of intracoronary nitroglycerin (Fig. 1). The patient's chest pain subsided instantly and echocardiography showed normal findings. She received oral administration of nitroglycerin, tramadol, and diltiazem in the ward. Five days after the surgery, the patient was discharged with a prescription for sublingual nitroglycerin. Despite two months' follow-up after discharge, there were no other problems, and her chest pain did not recur.

Coronary artery spasm caused by epinephrine has been reported very rarely $[2,3]$. It is known that ischemia and hypoxia reduce the threshold for coronary spasm as well as loss of autonomic control [2]. Blood catecholamine levels were not known in our case, but following the administration of epinephrine SC, there was an interval during which the increased BP was dramatically decreased over time, which can be assumed to be the cause of the coronary spasm.

In reviewing the adverse effects of epinephrine administration in the alert state $[2,3]$, it has been found that coronary artery spasm occurred in most cases when the patients complained of chest pain [2,3]. In order to treat coronary artery spasm, it is necessary to monitor and observe the patient continuously while controlling the BP with an IV nitrate infusion, IV morphine administration, and IV fluid infusion, and most patients have been discharged in a stable state after a few days $[2,3]$.

Although the patient in this case was injected with $1 \mathrm{mg}$ of epinephrine mixed with normal saline SC, which can be regarded as a relatively safe dose for clinical use [1,2], epinephrine can be absorbed rapidly through cervical mucus where there is increased blood flow [4]. It has been known that using epinephrine diluted with lidocaine rather than with normal saline decreases the incidence of ventricular arrhythmias due to the protective effect of lidocaine on epinephrine [5]. This patient was injected with epinephrine diluted with normal saline, so we assume that epinephrine with lidocaine could lower the potential toxicity for this patient.

We used esmolol for the treatment of epinephrine-induced tachyarrhythmia. Esmolol, a short-acting $\beta 1$-selective blocker, can reduce the risk of hypertension and coronary spasm due to unopposed $\alpha$-adrenergic stimulation, and it can be used to control catecholamine surges precisely [1].

In cases of hypotension or chest discomfort without bleeding following spinal anesthesia, most anesthesiologists consider the possibility of high-level neuraxial anesthesia. However, in cases in which epinephrine was administered for operative procedures during spinal anesthesia, most patient complaints of chest pain are caused by coronary artery spasm [3], and immediate confirmation of coronary spasm is important for proper management.

In conclusion, subcutaneous epinephrine injection under spinal anesthesia can induce coronary spasm. Early detection of coronary spasm through angiography and injection of intracoronary nitroglycerin could contribute to good prognosis. 


\section{References}

1. Bae JY, Woo CH, Kim SH, Kwak IS, Mun SH, Kim KM. Cardiovascular crisis after small dose local infiltration of epinephrine in patient with asymptomatic subarachnoid hemorrhage. Korean J Anesthesiol 2010; 59 Suppl: S53-7.

2. Karch SB. Coronary artery spasm induced by intravenous epinephrine overdose. Am J Emerg Med 1989; 7: 485-8.

3. Shaver KJ, Adams C, Weiss SJ. Acute myocardial infarction after administration of low-dose intravenous epinephrine for anaphylaxis. CJEM 2006; 8: 289-94.

4. Lee JY, Kim CH, Lee SJ, Jun NH, Park WK. Acute heart failure induced by a $\beta$-blocker after the local infiltration of epinephrine. Korean J Anesthesiol 2007; 52: 591-5.

5. Kim CJ, Kim JM, Jang YH, Shin YS. Cardiomyopathy after local infiltration or application of epinephrine for plastic surgery under general anesthesia. Korean J Anesthesiol 2009; 56: 725-8. 